\title{
VARIAÇÃO CROMOSSÔMICA NUMÉRICA EM Pennisetum
}

\author{
Chromosome number variation in Pennisetum
}

Lisete Chamma Davide', Vânia Helena Techio², Juliane Dornellas Nunes³, Antônio Vander Pereira ${ }^{4}$

\section{RESUMO}

Entre os acessos de Pennisetum da Coleção de Germoplasma da Embrapa Gado de Leite - Juiz de Fora, MG, avaliados em estudos anteriores, o BAG 55, capim-elefante cv. Kizozi, introduzido do Estado da Bahia e cuja origem genealógica é desconhecida, apresentou variação cromossômica numérica $(2 \mathrm{n}=54$ a 66 cromossomos) em diversas contagens, embora a sua identificação no Banco de Germoplasma como P. purpureum Schum. sugerisse que o mesmo apresentasse $2 \mathrm{n}=28$ cromossomos. Neste estudo foram feitas análises mitóticas comparativas entre este acesso com outros quatro representantes de capim-elefante (P. purpureum) e foram obtidas medições cromossômicas. Para o acesso BAG 55 também foram realizadas análises meióticas e de viabilidade de pólen. Os resultados das análises mitótica e meiótica confirmaram o número cromossômico desse acesso como sendo $2 \mathrm{n}=54$, indicando que não se trata de uma variedade de capim-elefante ( $P$. purpureum), mas de uma espécie selvagem de Pennisetum. As observações citogenéticas revelaram que há variações somente em nível somático, caracterizando-se como um caso de polissomatia e aneussomatia.

Termos para indexação: Pennisetum, citogenética, variação cromossômica numérica.

\begin{abstract}
Among the accessions of Pennisetum of the Germplasm Collection of Embrapa Gado de Leite - Juiz de Fora, MG evaluated in previous studies, BAG 55, elephant grass cv. Kizozi, introduced from the Bahia State and whose genealogical origin is unkown, presented chromosome number variation ( $2 n=54$ to 66 chromosomes) in several counts, although its identification in the Germplasm Bank as $P$. purpureum Schum. suggested that it presented $2 \mathrm{n}=28$ chromosomes. In this study comparative mitotic analysis among this accession with other four elephant grass ( $P$. purpureum) and chromosome measurements were performed. For the BAG 55 accession meiotic and pollen viability analysis were also accomplished. The results of the mitotic and meiotic analysis confirmed the chromosome number of the accession as $2 \mathrm{n}=54$, indicating that it is not an elephantgrass variety ( $P$. purpureum), but of a wild species of Pennisetum. The cytogenetic observations revealed that there are only variations in somatic level, as a case of polissomaty and aneussomaty.
\end{abstract}

Index terms: Pennisetum, cytogenetic, chromosome number variation.

\section{(Recebido em 3 de janeiro de 2005 e aprovado em 26 de janeiro de 2006)}

\section{INTRODUÇÃO}

Dentre os gêneros botânicos, Pennisetum caracteriza-se por sua complexidade e heterogeneidade devido aos inúmeros arranjos taxonômicos e ampla variabilidade genética descrita em aproximadamente 140 espécies. Entre elas destacam-se $P$. purpureum Schum. $(2 \mathrm{n}=4 \mathrm{x}=28)$ e P. $\operatorname{glaucum}(\mathrm{L}$.$) R. Br. (2 \mathrm{n}=2 \mathrm{x}=14)$ conhecidas como capim-elefante e milheto, respectivamente (BRUNKEN, 1977). Ambas espécies são importantes forrageiras cultivadas nas regiões tropicais do planeta e empregadas na obtenção de híbridos interespecíficos em programas de melhoramento genético.

Visando preservar a diversidade existente no gênero, a Embrapa Gado de Leite - Juiz de Fora-MG, constituiu um Banco de Germoplasma contendo acessos de Pennisetum e vários híbridos intra e interespecíficos. Avaliações morfológicas e citogenéticas têm contribuído para a identificação taxonômica e caracterização botânicaagronômica dos acessos dessa coleção.

Dentre os vários genótipos analisados (BARBOSA et al., 2003; TECHIO, 1998; TECHIO et al., 2002), observou-se que o acesso BAG 55 conhecido como capim-elefante baiano ou kizozi não apresentou o número cromossômico esperado, embora apresente características morfológicas similares às outras variedades de capim-elefante. $\mathrm{Na}$ análise citológica foi observada variação de 54 a 66 cromossomos em células de uma mesma raiz (TECHIO, 1998) deixando dúvida sobre a sua identidade botânica.

Techio (1998) sugeriu que a variação citogenética poderia ser atribuída à ocorrência de cromossomos $\mathrm{B}$ ou hibridação interespecífica seguida de mixoploidia.

\footnotetext{
Bióloga, Doutora em Ciências Florestais - Professora titular, Departamento de Biologia/DBI - Universidade Federal de Lavras/UFLA - Cx. P. 3037 37200-000 - Lavras, MG __Icdavide@ufla.br

${ }^{2}$ Bióloga, Doutora em Genética e Melhoramento de Plantas - Professora, Curso de Ciências Biológicas - Universidade do Contestado - Concórdia, SC vht@uncnet.br

${ }^{3}$ Engenheira Agrônoma - Doutoranda em Genética e Melhoramento de Plantas - Universidade Federal de Lavras/UFLA - Cx. P. 3037 - 37.200-000 Lavras, MG - judornellas@bol.com.br

${ }^{4}$ Engenheiro Agrônomo, Doutor em Genética Vegetal - Pesquisador da Embrapa Gado de Leite - Juiz de Fora, MG - avanderp@ @npgl.embrapa.br
} 
A mixoploidia, ocasionada por eliminações cromossômicas, é comum em híbridos interespecíficos e intergenéricos e também já foi descrita para híbridos de Pennisetum (GILDENHUYS \& BRIX, 1964; RAMAN \& KRISHASWAMY, citados por JAUHAR, 1981). Recentemente, Abreu (2002) relatou perdas cromossômicas em híbridos de milheto e capim-elefante submetidos a diferentes concentrações e tempos de exposição a substâncias antimitóticas.

Já em relação à ocorrência de cromossomos $\mathrm{B}$ em Pennisetum, Pantulu (1960) foi o primeiro a descrevê-los em $P$. typhoides ( $\sin$. P. glaucum), registrando uma variação de 1 a 3 em relação ao complemento normal de $2 n=14$. Para esta mesma espécie, Jones \& Rees (1982) relataram até 8 cromossomos B e citaram $P$. squamulatum e $P$. orientale com até 2 a 3 cromossomos B, respectivamente. Em $P$. violaceum, Khalfallah et al. (1993) descreveram cromossomos $\mathrm{B}$ do tipo metacêntrico e número variando de 1 a 4.

A instabilidade no número cromossômico é um fato interessante a ser investigado em citogenética, pois sua ocorrência é considerada uma anormalidade. Em vista disso e também pela importância do acesso BAG 55 no Banco de Germoplasma e no programa de melhoramento genético do capim-elefante e considerando que não foram encontrados relatos semelhantes na literatura para a espécie $P$. purpureum, o presente trabalho teve por objetivo avaliar citogeneticamente o acesso BAG 55 e fazer a comparação das medições dos cromossomos deste acesso com outros de $P$. purpureum, a fim de explicar a variação cromossômica e contribuir para esclarecer a sua identificação botânica.

\section{MATERIAL E MÉTODOS}

Foi avaliado o número cromossômico de cinco acessos de P. purpureum (BAGs 27, 45, 46, 55 e 103) do Banco Ativo de Germoplasma de Plantas Forrageiras da Embrapa Gado de Leite - Juiz de Fora-MG.

A determinação do número de cromossomos foi feita por meio de análises em metáfases obtidas de meristemas radiculares procedentes do enraizamento de estacas com 2 a 3 nós de plantas adultas.

Para obtenção das metáfases mitóticas foi utilizada a técnica de esmagamento e coloração adaptada por Techio et al. (2002) para espécies de Pennisetum, com tratamento prévio de sincronização do ciclo celular, seguindo recomendações de Lee et al. (1997). Para o BAG 55 também foi realizada análise meiótica utilizando-se o protocolo convencional com corante carmim propiônico $0,5 \%$. A viabilidade do pólen foi determinada por meio de coloração com os corantes de Alexander, orceína acética $1 \%$ e carmim propiônico $2 \%$

As fotomicrografias foram feitas em fotomicroscópio Olympus BX60, utilizando-se filmes Kodak.

Nas metáfases mitóticas, foram avaliados: comprimento do braço maior - BL; comprimento do braço menor $-\mathrm{BC}$; comprimento total do cromossomo $-\mathrm{Cti}=\mathrm{BL}$ $+\mathrm{BC}$; a relação de braços $-\mathrm{RB}=\mathrm{BL} / \mathrm{BC}$, o comprimento total do lote diplóide $-\mathrm{CTLD}=\mathrm{S}$ Cti e o comprimento relativo $-\mathrm{CR}=\mathrm{Cti} / \mathrm{CTLD} \mathrm{X} 100$. A classificação dos cromossomos quanto à posição do centrômero foi feita segundo a nomenclatura de Levan et al. (1964).

Foi efetuada a análise de variância para o comprimento total do lote diplóide (CTLD) entre os acessos, seguido do teste de Scott \& Knott (1974).

\section{RESULTADOS E DISCUSSÃO}

Para quatro dos acessos analisados (BAGs 27, 45, 46 e 103) foram confirmadas metáfases com $2 n=28$ cromossomos (Figura 1), já amplamente descrito na literatura para P. purpureum (BARBOSA et al., 2003; BRUNKEN, 1977; BURTON, 1942; MANARA, 1973; TECHIO et al., 2002). Para o acesso BAG 55 foi observada variação numérica de $2 n=54,56,60,61$ e 66 cromossomos em metáfases da mesma região meristemática (Figura 2), sendo 2 n=54 o número encontrado com maior freqüência.

Os quatro acessos com $2 \mathrm{n}=28$ foram agrupados pelo teste de Scott-Knott revelando que não existem diferenças significativas entre eles no que se refere ao comprimento total do lote diplóide (CTLD), cujo valor médio foi de $51,19 \mathrm{~mm}$. O mesmo não ocorreu com o BAG 55, que apresentou valor médio para o CTLD de 99,298 mm (Tabela 1).

O complemento cromossômico dos acessos de capim-elefante caracterizou-se pela alta frequência de cromossomos metacêntricos (Tabela 2), o que contribui para acentuar a similaridade entre os cariótipos, sugerindo uma tendência de cariótipo simétrico, de acordo com a classificação de Stebbins (1958). Por apresentar cromossomos com tamanho reduzido (Tabela 3) e por utilizarmos coloração convencional, não foi possível estabelecer a classificação dos cromossomos do BAG 55. 


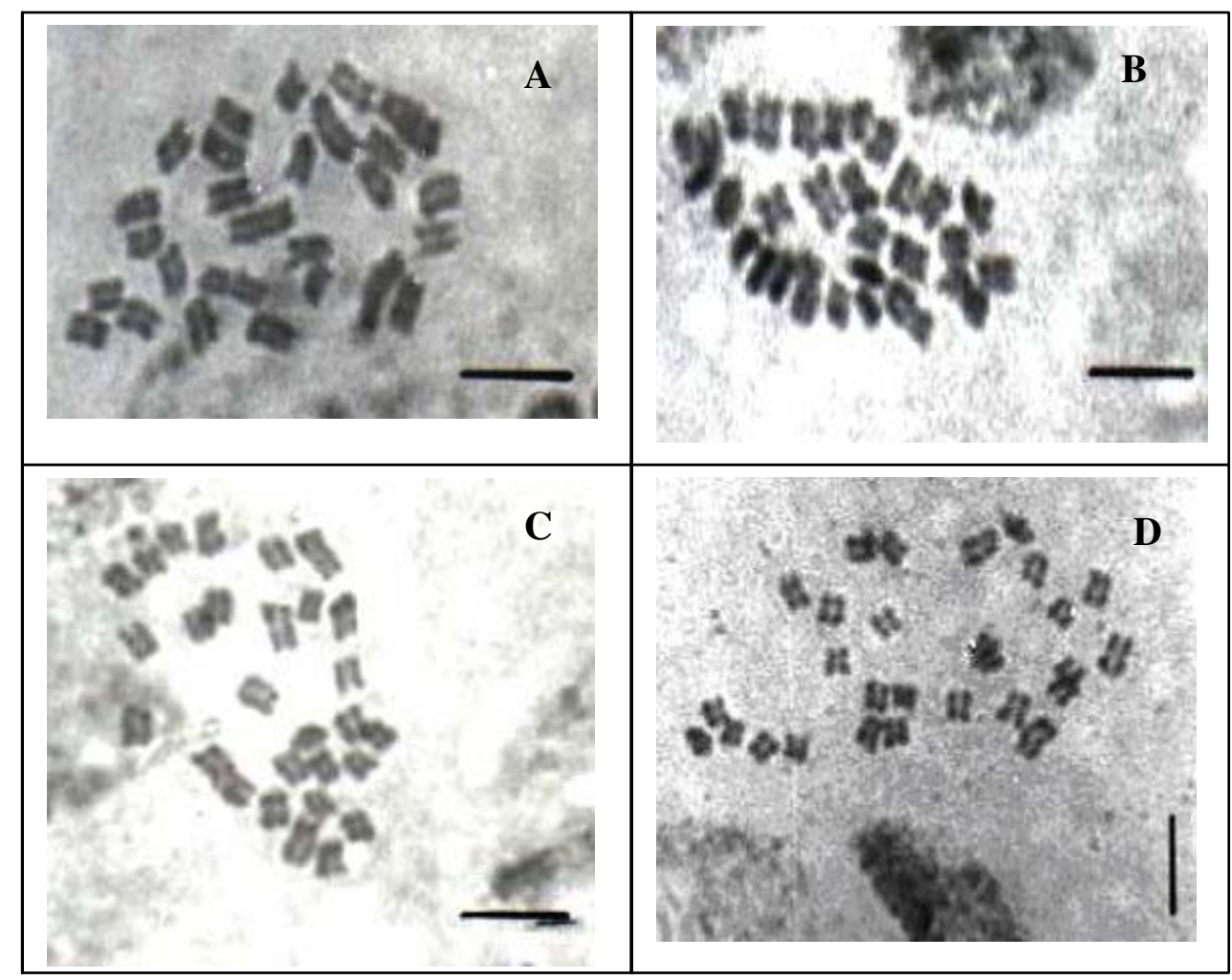

FIGURA 1-Metáfases mitóticas. A. BAG 27; B. BAG 45; C. BAG 46 e D. BAG 103 (P. purpureum 2n=28 cromossomos). As barras representam $5 \mu \mathrm{m}$.

Nas análises meióticas do BAG 55 foram observadas diacineses e metáfases com 27 bivalentes (Figura 3) e também foram encontrados meiócitos com anormalidades, tais como: aderências cromossômicas, metáfases com ascensão precoce e micronúcleos. Ainda assim, a viabilidade do pólen foi de $99 \%$ em testes com os corantes de Alexander, orceína acética e carmim propiônico.

Os resultados das análises mitótica e meiótica confirmam o número cromossômico somático desse acesso como sendo $2 \mathrm{n}=54$, corroborando com Techio (1998), de que não se trata de uma variedade de capimelefante ( $P$. purpureum), mas sim uma espécie selvagem de Pennisetum.

As observações citogenéticas revelaram que há variações somente em nível somático, o que é mencionado por Nirmala \& Rao (1996) como sendo polissomatia e aneussomatia, expressões referidas à euploidia e aneuploidia em células somáticas, respectivamente. As principais causas dessas variações são a endopoliploidia, redução genômica, anormalidades no fuso, sincício e semigamia, que podem ter como resultados a não disjunção, redução somática, eliminação cromossômica, fusos múltiplos, fracionamento do complemento cromossômico, duplicação genômica (NIRMALA \& RAO, 1996). Esses eventos são amplamente discutidos na literatura, porém os mecanismos bioquímicos e moleculares permanecem desconhecidos e ainda pouco estudados. 


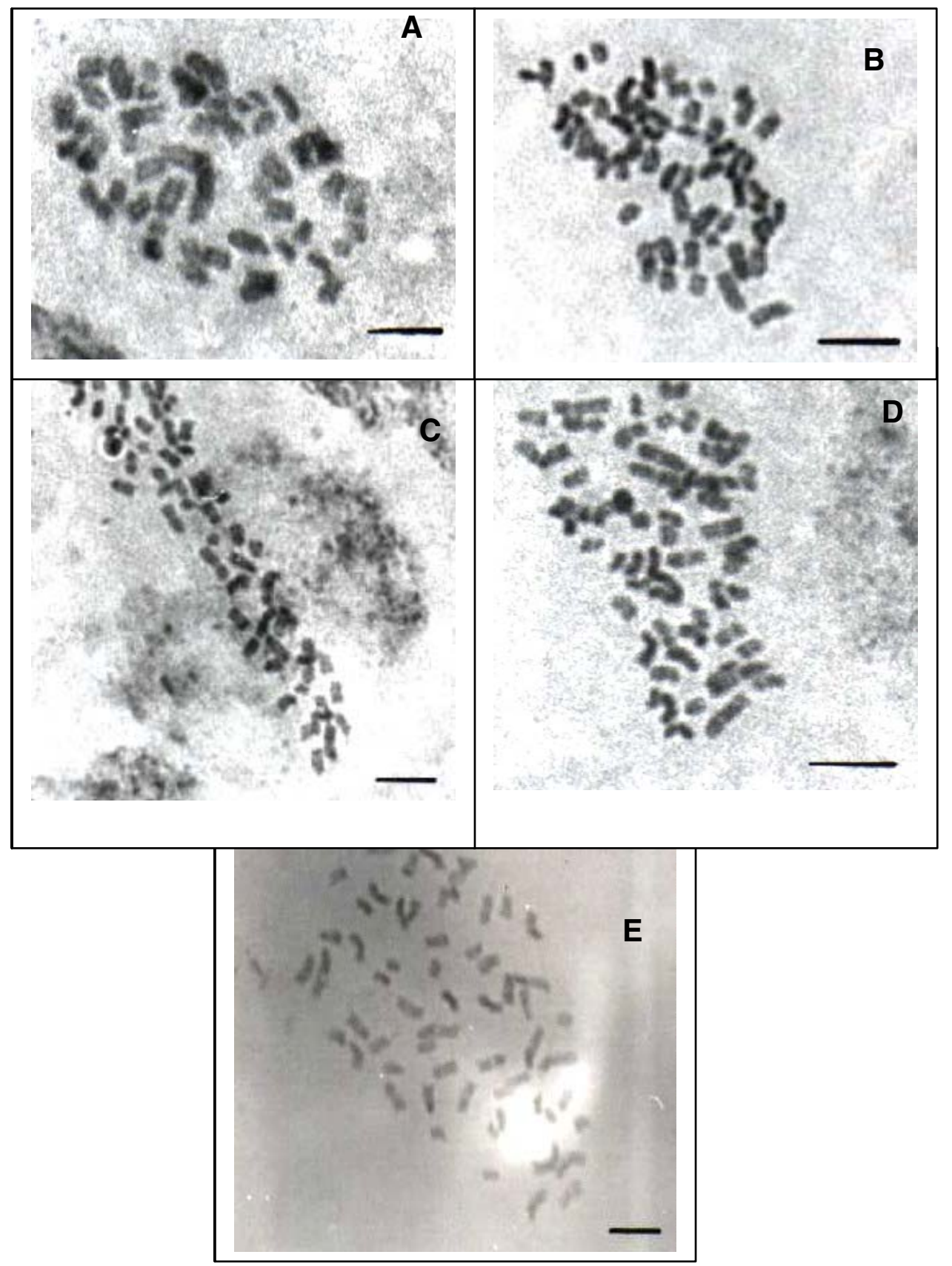

FIGURA 2 - Metáfases mitóticas do acesso BAG 55. A. 2n=54 cromossomos; B.2n=56 cromossomos; C. 2n=60 cromossomos; D. 2n=61 cromossomos e E. $2 \mathrm{n}=66$ cromossomos. As barras representam $5 \mu \mathrm{m}$. 
TABELA 1 - Comprimento total do lote diplóide, em micrômetros, para os acessos BAGs 27, 45, 46, 103 e 55 e agrupamento baseado no teste de Scott-Knott, ao nível de significância de 5\%.

\begin{tabular}{ll}
\hline Tratamentos & Médias \\
\hline BAG 103 & 46,460000 a1 \\
BAG 46 & 48,923333 a1 \\
BAG 45 & 50,123333 a1 \\
BAG27 & 59,270000 a1 \\
BAG 55 & 99,298000 a2 \\
\hline
\end{tabular}

TABELA 2 - Valores médios, em micrômetros $(\mu \mathrm{m})$, das variáveis cromossômicas ( $\mathrm{Cti}=$ comprimento total do cromossomo; $\mathrm{CR}=$ comprimento relativo e $\mathrm{CTLD}=$ comprimento total do lote diplóide) dos acessos de capim-elefante (BAGs 27, 45, 46 e 103). $(C=$ cromossomo; $\mathrm{Cl}=$ classificação do cromossomo em relação à posição do centrômero, sendo $\mathrm{m}=$ metacêntrico; $\mathrm{sm}=$ submetacêntrico).

\begin{tabular}{|c|c|c|c|c|c|c|c|}
\hline $\mathbf{C}$ & Cti & CR & Cl & $\mathbf{C}$ & Cti & CR & Cl \\
\hline 1 & 2,63 & 5,11 & $\mathrm{~m}$ & 15 & 1,71 & 3,32 & $\mathrm{~m}$ \\
\hline 2 & 2,52 & 4.88 & $\mathrm{~m}$ & 16 & 1,71 & 3,32 & $\mathrm{~m}$ \\
\hline 3 & 2,34 & 4,54 & $\mathrm{~m}$ & 17 & 1,71 & 3,32 & $\mathrm{~m}$ \\
\hline 4 & 2,30 & 4,46 & $\mathrm{~m}$ & 18 & 1,69 & 3,26 & $\mathrm{~m}$ \\
\hline 5 & 2,22 & 4,31 & $\mathrm{sm}$ & 19 & 1,69 & 3,26 & $\mathrm{~m}$ \\
\hline 6 & 2,11 & 4,09 & $\mathrm{~m}$ & 20 & 1,69 & 3,26 & $\mathrm{~m}$ \\
\hline 7 & 2,08 & 4,03 & $\mathrm{sm}$ & 21 & 1,69 & 3,26 & $\mathrm{~m}$ \\
\hline 8 & 2,02 & 3,92 & $\mathrm{~m}$ & 22 & 1,63 & 3,15 & $\mathrm{~m}$ \\
\hline 9 & 1,88 & 3,66 & $\mathrm{~m}$ & 23 & 1,60 & 3,10 & $\mathrm{~m}$ \\
\hline 10 & 1,85 & 3,60 & $\mathrm{~m}$ & 24 & 1,55 & 2,99 & $\mathrm{~m}$ \\
\hline 11 & 1,82 & 3,54 & $\mathrm{~m}$ & 25 & 1,52 & 2,94 & $\mathrm{~m}$ \\
\hline 12 & 1,80 & 3,48 & $\mathrm{~m}$ & 26 & 1,46 & 2,83 & $\mathrm{~m}$ \\
\hline 13 & 1,74 & 3,37 & $\mathrm{~m}$ & 27 & 1,41 & 2,73 & $\mathrm{~m}$ \\
\hline \multirow[t]{2}{*}{14} & 1,74 & 3,37 & $\mathrm{~m}$ & 28 & 1,38 & 2,68 & $\mathrm{~m}$ \\
\hline & & & & CTLD & & $51,54 \mu \mathrm{m}$ & \\
\hline
\end{tabular}

Ciênc. agrotec., Lavras, v. 31, n. 2, p. 398-405, mar./abr., 2007 
TABELA 3 - Valores médios, em micrômetros $(\mu \mathrm{m})$, das variáveis cromossômicas $(\mathrm{Cti}=$ comprimento total do cromossomo; $\mathrm{CR}=$ comprimento relativo e $\mathrm{CTLD}=$ comprimento total do lote diplóide) do acesso BAG 55. $\mathrm{C}=$ cromossomo.

\begin{tabular}{|c|c|c|c|c|c|c|c|c|c|c|c|c|}
\hline & \multicolumn{2}{|c|}{$2 n=54$} & \multicolumn{2}{|c|}{$2 n=56$} & \multicolumn{2}{|c|}{$2 n=60$} & \multicolumn{2}{|c|}{$2 n=61$} & \multicolumn{2}{|c|}{$2 n=66$} & \multicolumn{2}{|c|}{ Média } \\
\hline $\mathbf{C}$ & Cti & CR & Cti & CR & Cti & CR & Cti & CR & Cti & $\mathbf{C R}$ & Cti & $\mathbf{C R}$ \\
\hline 1 & 3,33 & 3,25 & 2,66 & 3,15 & 2,53 & 3,02 & 2,66 & 2,25 & 2,66 & 2,45 & 2,76 & 2,82 \\
\hline 2 & 3,00 & 2,92 & 2,33 & 2,76 & 2,00 & 2,38 & 2,66 & 2,25 & 2,66 & 2,45 & 2,53 & 2,55 \\
\hline 3 & 3,00 & 2,92 & 2,33 & 2,76 & 2,00 & 2,38 & 2,66 & 2,25 & 2,66 & 2,45 & 2,53 & 2,55 \\
\hline 4 & 2,66 & 2,59 & 2,33 & 2,76 & 2,00 & 2,38 & 2,66 & 2,25 & 2,66 & 2,45 & 2,46 & 2,48 \\
\hline 5 & 2,66 & 2,59 & 2,00 & 2,37 & 2,00 & 2,38 & 2,66 & 2,25 & 2,33 & 2,15 & 2,33 & 2,34 \\
\hline 6 & 2,66 & 2,59 & 2,00 & 2,37 & 2,00 & 2,38 & 2,66 & 2,25 & 2,33 & 2,15 & 2,33 & 2,34 \\
\hline 7 & 2,66 & 2,59 & 2,00 & 2,37 & 2,00 & 2,38 & 2,66 & 2,25 & 2,33 & 2,15 & 2,33 & 2,34 \\
\hline 8 & 2,66 & 2,59 & 2,00 & 2,37 & 2,00 & 2,38 & 2,66 & 2,25 & 2,00 & 1,84 & 2,26 & 2,28 \\
\hline 9 & 2,66 & 2,59 & 2,00 & 2,37 & 2,00 & 2,38 & 2,66 & 2,25 & 2,00 & 1,84 & 2,26 & 2,28 \\
\hline 10 & 2,53 & 2,47 & 2,00 & 2,37 & 1,66 & 1,98 & 2,33 & 1,97 & 2,00 & 1,84 & 2,10 & 2,12 \\
\hline 11 & 2,53 & 2,47 & 2,00 & 2,37 & 1,66 & 1,98 & 2,33 & 1,97 & 2,00 & 1,84 & 2,10 & 2,12 \\
\hline 12 & 2,33 & 2,27 & 2,00 & 2,37 & 1,66 & 1,98 & 2,33 & 1,97 & 2,00 & 1,84 & 2,06 & 2,12 \\
\hline 13 & 2,33 & 2,27 & 1,66 & 1,97 & 1,66 & 1,98 & 2,33 & 1,97 & 2,00 & 1,84 & 1,99 & 2,04 \\
\hline 14 & 2,33 & 2,27 & 1,66 & 1,97 & 1,66 & 1,98 & 2,00 & 1,69 & 2,00 & 1,84 & 1,93 & 1,99 \\
\hline 15 & 2,33 & 2,27 & 1,66 & 1,97 & 1,66 & 1,98 & 2,00 & 1,69 & 2,00 & 1,84 & 1,93 & 1,99 \\
\hline 16 & 2,33 & 2,27 & 1,66 & 1,97 & 1,66 & 1,98 & 2,00 & 1,69 & 2,00 & 1,84 & 1,93 & 1,99 \\
\hline 17 & 2,33 & 2,27 & 1,66 & 1,97 & 1,66 & 1,98 & 2,00 & 1,69 & 2,00 & 1,84 & 1,93 & 1,99 \\
\hline 18 & 2,00 & 1,95 & 1,66 & 1,97 & 1,66 & 1,98 & 2,00 & 1,69 & 2,00 & 1,84 & 1,86 & 1,88 \\
\hline 19 & 2,00 & 1,95 & 1,66 & 1,97 & 1,66 & 1,98 & 2,00 & 1,69 & 2,00 & 1,84 & 1,86 & 1,88 \\
\hline 20 & 2,00 & 1,95 & 1,66 & 1,97 & 1,46 & 1,74 & 2,00 & 1,69 & 2,00 & 1,84 & 1,82 & 1,83 \\
\hline 21 & 2,00 & 1,95 & 1,66 & 1,97 & 1,46 & 1,74 & 2,00 & 1,69 & 2,00 & 1,84 & 1,82 & 1,83 \\
\hline 22 & 2,00 & 1,95 & 1,66 & 1,97 & 1,46 & 1,74 & 2,00 & 1,69 & 2,00 & 1,84 & 1,82 & 1,83 \\
\hline 23 & 2,00 & 1,95 & 1,66 & 1,97 & 1,46 & 1,74 & 2,00 & 1,69 & 2,00 & 1,84 & 1,82 & 1,83 \\
\hline 24 & 2,00 & 1,95 & 1,66 & 1,97 & 1,33 & 1,58 & 2,00 & 1,69 & 2,00 & 1,84 & 1,79 & 1,80 \\
\hline 25 & 2,00 & 1,95 & 1,33 & 1,57 & 1,33 & 1,58 & 2,00 & 1,69 & 2,00 & 1,84 & 1,73 & 1,72 \\
\hline 26 & 2,00 & 1,95 & 1,33 & 1,57 & 1,33 & 1,58 & 2,00 & 1,69 & 1,66 & 1,53 & 1,66 & 1,66 \\
\hline 27 & 2,00 & 1,95 & 1,33 & 1,57 & 1,33 & 1,58 & 2,00 & 1,69 & 1,66 & 1,53 & 1,66 & 1,66 \\
\hline 28 & 2,00 & 1,95 & 1,33 & 1,57 & 1,33 & 1,58 & 2,00 & 1,69 & 1,66 & 1,53 & 1,66 & 1,66 \\
\hline 29 & 1,86 & 1,81 & 1,33 & 1,57 & 1,33 & 1,58 & 2,00 & 1,69 & 1,66 & 1,53 & 1,63 & 1,63 \\
\hline 30 & 1,86 & 1,81 & 1,33 & 1,57 & 1,33 & 1,58 & 2,00 & 1,69 & 1,66 & 1,53 & 1,63 & 1,63 \\
\hline 31 & 1,86 & 1,81 & 1,33 & 1,57 & 1,33 & 1,58 & 2,00 & 1,69 & 1,66 & 1,53 & 1,63 & 1,63 \\
\hline 32 & 1,86 & 1,81 & 1,33 & 1,57 & 1,33 & 1,58 & 2,00 & 1,69 & 1,66 & 1,53 & 1,63 & 1,63 \\
\hline 33 & 1,86 & 1,81 & 1,33 & 1,57 & 1,33 & 1,58 & 2,00 & 1,69 & 1,66 & 1,53 & 1,63 & 1,63 \\
\hline 34 & 1,86 & 1,81 & 1,33 & 1,57 & 1,33 & 1,58 & 2,00 & 1,69 & 1,66 & 1,53 & 1,63 & 1,63 \\
\hline 35 & 1,86 & 1,81 & 1,33 & 1,57 & 1,33 & 1,58 & 2,00 & 1,69 & 1,66 & 1,53 & 1,63 & 1,63 \\
\hline 36 & 1,66 & 1,62 & 1,33 & 1,57 & 1,33 & 1,58 & 2,00 & 1,69 & 1,66 & 1,53 & 1,59 & 1,59 \\
\hline 37 & 1,66 & 1,62 & 1,33 & 1,57 & 1,33 & 1,58 & 1,66 & 1,40 & 1,33 & 1,22 & 1,46 & 1,47 \\
\hline 38 & 1,66 & 1,62 & 1,33 & 1,57 & 1,33 & 1,58 & 1,66 & 1,40 & 1,33 & 1,22 & 1,46 & 1,47 \\
\hline 39 & 1,66 & 1,62 & 1,33 & 1,57 & 1,33 & 1,58 & 1,66 & 1,40 & 1,33 & 1,22 & 1,46 & 1,47 \\
\hline 40 & 1,66 & 1,62 & 1,33 & 1,57 & 1,33 & 1,58 & 1,66 & 1,40 & 1,33 & 1,22 & 1,46 & 1,47 \\
\hline 41 & 1,66 & 1,62 & 1,33 & 1,57 & 1,33 & 1,58 & 1,66 & 1,40 & 1,33 & 1,22 & 1,46 & 1,47 \\
\hline 42 & 1,66 & 1,62 & 1,33 & 1,57 & 1,20 & 1,43 & 1,66 & 1,40 & 1,33 & 1,22 & 1,43 & 1,44 \\
\hline 43 & 1,66 & 1,62 & 1,33 & 1,57 & 1,20 & 1,43 & 1,66 & 1,40 & 1,33 & 1,22 & 1,43 & 1,44 \\
\hline 44 & 1,66 & 1,62 & 1,33 & 1,57 & 1,20 & 1,43 & 1,66 & 1,40 & 1,33 & 1,22 & 1,43 & 1,44 \\
\hline 45 & 1,66 & 1,62 & 1,33 & 1,57 & 1,20 & 1,43 & 1,66 & 1,40 & 1,33 & 1,22 & 1,43 & 1,44 \\
\hline 46 & 1,33 & 1,29 & 1,33 & 1,57 & 1,20 & 1,43 & 1,66 & 1,40 & 1,33 & 1,22 & 1,43 & 1,38 \\
\hline
\end{tabular}

Continua... 
TABELA 3-Continuação...

\begin{tabular}{|c|c|c|c|c|c|c|c|c|c|c|c|c|}
\hline $\mathrm{C}$ & Cti & CR & Cti & CR & Cti & CR & Cti & CR & Cti & CR & Cti & CR \\
\hline 47 & 1,33 & 1,29 & 1,00 & 1,18 & 1,00 & 1,19 & 1,66 & 1,40 & 1,33 & 1,22 & 1,26 & 1,25 \\
\hline 48 & 1,33 & 1,29 & 1,00 & 1,18 & 1,00 & 1,19 & 1,66 & 1,40 & 1,33 & 1,22 & 1,26 & 1,25 \\
\hline 49 & 1,33 & 1,29 & 1,00 & 1,18 & 1,00 & 1,19 & 1,66 & 1,40 & 1,33 & 1,22 & 1,26 & 1,25 \\
\hline 50 & 1,33 & 1,29 & 1,00 & 1,18 & 1,00 & 1,19 & 1,66 & 1,40 & 1,33 & 1,22 & 1,26 & 1,25 \\
\hline 51 & 1,33 & 1,29 & 1,00 & 1,18 & 1,00 & 1,19 & 1,66 & 1,40 & 1,33 & 1,22 & 1,26 & 1,25 \\
\hline 52 & 1,33 & 1,29 & 1,00 & 1,18 & 1,00 & 1,19 & 1,66 & 1,40 & 1,33 & 1,22 & 1,26 & 1,25 \\
\hline 53 & 1,20 & 1,17 & 1,00 & 1,18 & 1,00 & 1,19 & 1,66 & 1,40 & 1,33 & 1,22 & 1,23 & 1,23 \\
\hline 54 & 1,00 & 0,97 & 0,80 & 0,94 & 1,00 & 1,19 & 1,33 & 1,12 & 1,33 & 1,22 & 1,09 & 1,08 \\
\hline 55 & & & 0,80 & 0,94 & 0,80 & 0,95 & 1,33 & 1,12 & 1,33 & 1,22 & 1,06 & 1,05 \\
\hline 56 & & & 0,80 & 0,94 & 0,80 & 0,95 & 1,33 & 1,12 & 1,20 & 1,10 & 1,03 & 1,02 \\
\hline 57 & & & & & 0,80 & 0,95 & 1,33 & 1,12 & 1,00 & 0,92 & 1,04 & 0,99 \\
\hline 58 & & & & & 0,80 & 0,95 & 1,33 & 1,12 & 1,00 & 0,92 & 1,04 & 0,99 \\
\hline 59 & & & & & 0,80 & 0,95 & 1,33 & 1,12 & 1,00 & 0,92 & 1,04 & 0,99 \\
\hline 60 & & & & & 0,80 & 0,95 & 1,33 & 1,12 & 1,00 & 0,92 & 1,04 & 0,99 \\
\hline 61 & & & & & & & 1,00 & 0,84 & 1,00 & 0,92 & 1,00 & 0,88 \\
\hline 62 & & & & & & & & & 1,00 & 0,92 & 1,00 & 0,92 \\
\hline 63 & & & & & & & & & 1,00 & 0,92 & 1,00 & 0,92 \\
\hline 64 & & & & & & & & & 1,00 & 0,92 & 1,00 & 0,92 \\
\hline 65 & & & & & & & & & 1,00 & 0,92 & 1,00 & 0,92 \\
\hline 66 & & & & & & & & & 1,00 & 0,92 & 1,00 & 0,92 \\
\hline CTLD & 102,40 & & 84,23 & & 83,71 & & 117,79 & & 108,36 & & 124,12 & \\
\hline
\end{tabular}

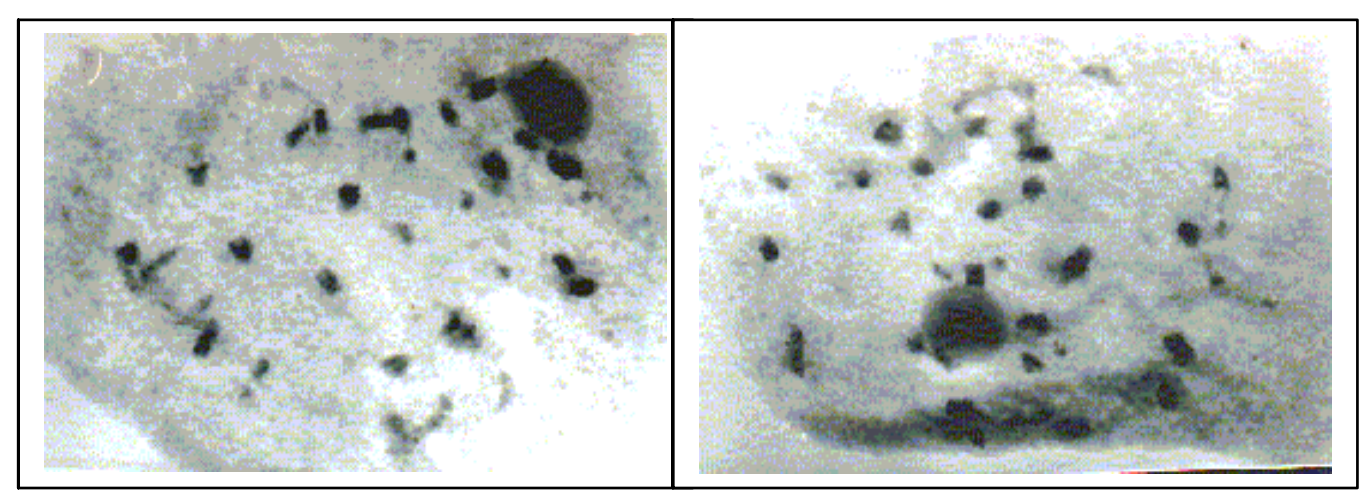

FIGURA 3 - BAG 55. Diacineses com 27 bivalentes.

\section{CONCLUSÕES}

Os resultados das análises mitótica e meiótica confirmaram o número cromossômico somático 54 para o acesso BAG 55, indicando tratar-se de uma espécie selvagem de Pennisetum. As observações citogenéticas revelaram que há variações somente em nível somático, caracterizando-se como um caso de polissomatia e aneussomatia.

\section{AGRADECIMENTOS}

Os autores agradecem à FAPEMIG, pelo auxílio financeiro à pesquisa e ao PIBIC/CNPq, pela concessão de bolsa de iniciação científica. 


\section{REFERÊNCIAS BIBLIOGRÁFICAS}

ABREU, J. C. Mixoploidia em híbridos de capim-elefante $\mathbf{x}$ milheto tratados com agentes antimitóticos. 2002. 119 p. Tese (Doutorado em Genética e Melhoramento de Plantas) - Universidade Federal de Lavras, Lavras, 2002.

BARBOSA, S.; DAVIDE, L. C.; PEREIRA, A. V. Citogenética de híbridos entre Pennisetum purpureum Schumach. e Pennisetum glaucum L. e seus genitores. Ciência e Agrotecnologia, Lavras, v. 27, n. 1, p. 26-35, 2003.

BRUNKEN, J. N. A systematic study of Pennisetum Sect. Pennisetum (Gramineae). American Journal of Botany, New York, v. 64, n. 2, p. 161-176, 1977.

BURTON, G. W. A cytological study of some species in the Tribe Paniceae. American Journal of Botany, New York, v. 29, p. 355-361, 1942.

GILDENHUYS, P.; BRIX, K. Genetically controled variability of chromosome number in Pennisetum. Heredity, Washington, v. 19, p. 533-542, 1964.

JAUHAR, P. P. Cytogenetics of pearl millet. Advances in Agronomy, New York, v. 34, p. 407-479, 1981.

JONES, R. N.; REES, H. B chromosomes. New York: Academic, 1982. $266 \mathrm{p}$.

KHALFALLAH, N.; SARR, A.; SILJAK-YAKOVLEV, S. Karyological study of some cultivated and wild stocks of pearl millet from Africa (Pennisetum typhoides Stapf et Hubb. and $P$. violaceum (Lam.) L. Rich.). Caryologia, Pisa, v. 46, p. $127-138,1993$.

LEE, J.; ARUMUGANATHAN, K.; YEN, Y.; KAEPPLER, S.; KAEPPLER, H.; BAENZIGER, P. S. Root tip cell-cycle synchronization and metaphase-chromosome isolation suitable for flow sorting in common wheat (Triticum aestivum L.). Genome, Otawa, v. 40, p. 633-638, 1997.

LEVAN, A.; FEDGA, K.; SOUBERG, A. A. Nomenclature for centromeric position on chromosomes. Hereditas, Landskrona, v. 52, p. 201-220, 1964.

MANARA, N. T. F. Citogenética de variedades de capim elefante (Pennisetum purpureum Schum.). 1973. 63 p. Dissertação (Mestrado em Agronomia) - Escola Superior de Agricultura Luiz de Queiroz, Piracicaba, 1973.

NIRMALA, A.; RAO, P. N. Genesis of chromosome numerical mosaicism in higher plants. The Nucleus, Lahore, v. 39, p. 151-175, 1996.

PANTULU, J. V. Accessory chromosomes in P. typhoides. Current Science, Calcutta, v. 29, p. 28-29, 1960.

SCOTT, A. J.; KNOTT, M. A cluster analysis method for grouping means in the analysis of variance. Biometrics, Releigh, v. 30, p. 507-512, 1974.

STEBBINS, G. L. Longevity, habitat e release of genetic variability in the higher plants. Cold Spring Harbor Symposia Quantitative Biology, [S.1.], v. 23, p. 365-378, 1958.

TECHIO, V. H. Citotaxonomia de algumas espécies e de híbridos interespecíficos de Pennisetum. 1998. 112 p. Dissertação (Mestrado em Genética e Melhoramento de Plantas) - Universidade Federal de Lavras, Lavras, 1998.

TECHIO, V. H.; DAVIDE, L. C.; PEREIRA, A. V.; BEARZOTI, E. Cytotaxonomy of some species and of interspecific hybrids of Pennisetum (Poaceae, Poales). Genetics and Molecular Biology, Ribeirão Preto, v. 25, n. 2, p. 203-209, 2002. 